\title{
Combat Performance Evaluation and the Analysis Combat Requirements of Amphibious Armored Vehicles
}

\author{
Chunxu Li ${ }^{1, a}$, Zhanjun Niu ${ }^{2, b}$, Baozhan Qin ${ }^{3, c}$, Zhenggen $\mathrm{Yu}^{4, \mathrm{~d}}$, \\ Xuesong Tang ${ }^{5, e}$, Xiaobing $\mathrm{Lu}^{6, \mathrm{f}}$ \\ $1,2,3,4,5,6$ changchun engineering technology college, Changchun, 130117, China \\ a,b,c,d,e,femail: 13547021@qq.com
}

\begin{abstract}
Keywords: amphibious armored vehicles,Operational performance, Demand,Grey fixed weight clustering,Triangular whitenization weight function
\end{abstract}

\begin{abstract}
The amphibious armored vehicles will be the future integration across the sea island, especially the battle equipment of emergency operations, based on grey fixed weight clustering and triangle whitenization weight function, put forward to evaluate performance of amphibious armored vehicle combat general steps. In the case of a certain type of land and water tanks, the operational performance is analyzed before and after modification, and put forward the scientific Suggestions for its future improvement. According to the characteristics of the battlefield environment under the background of actual combat, based on the bicycle to combat effectiveness evaluation, through introducing the battlefield environment and battlefield viability index, improved ADC model is put forward, and the integrated use of analytic hierarchy process (ahp) and the power index method and other methods to quantify the corresponding child model parameters, adopt the method of combining qualitative analysis with quantitative analysis, the implementation of operational effectiveness evaluation of a certain type of amphibious armored vehicles. And through examples to prove the effectiveness of the model proposed, have very strong pertinence and guidance.
\end{abstract}

\section{Introduction}

Amphibious armored vehicle integration across the sea in the future will play an important role in landing operations. Of amphibious armored vehicles to improve the operational performance, under the existing conditions is converted to a less money, a good way to take a short, quick effect. For example, a certain type of amphibious tank modified bike costs rose, and the operational performance is from "poor" to "good" class. Actually serving for weapons and equipment to be modified and army customary practice in many countries, such as Russian forces to informatization transformation of main battle tanks, U.S. strategic bombers loading new electronic radar, gives the ability to combat all increased significantly. For amphibious armored vehicles to be modified, usually in front of the modified according to its operational performance index (or are expected to realize value), the realization of the value to evaluate operational performance before and after modification analysis, to determine the need to improve, indicate the direction for equipment improvement.

The ADC method is American industry weapon system effectiveness put forward by the advisory committee (WESICA) method for weapon system effectiveness evaluation. The operational effectiveness of A certain type of amphibious armored vehicles refers to the vehicle system under the condition of combat, A measure of the degree, complete certain tasks is the availability of A certain type of amphibious armored vehicles system (A), reliability (D), operational capability (C) function. Theoretical analysis method based on the ideal weapon system, according to the characteristics of amphibious battlefield environment under the condition of considering against the operational effectiveness of a certain type of li habitat armored vehicles, to improve the basic model of ADC, combined with analytic hierarchy process (ahp) and the ability of power index method to quantitatively describe indicators for qualitative assessment. 


\section{Evaluation methods and steps}

\section{Evaluation Methods.}

For amphibious armored vehicles operational performance evaluation, the index system and index to realize the value of the scope of classification, can be set in advance by qualitative analysis and the appropriate calculation, the main problem is detecting index value which belong to the category, therefore USES the grey class triangular whitenization weight function clustering method for evaluation. At the same time, due to the diversity of the indicators of performance operational performance, and have different meanings, different dimension, the number of indicators to achieve value disparity is very big, in this case for each cluster index empowerment in advance.

B.Evaluation Steps

Operational performance before and after the amphibious armored vehicles refitted grey clustering evaluation, can be seen as $\mathrm{n}$ objects, the evaluation index, $\mathrm{s}$ a $\mathrm{m}$ a different class, according to the object I about index $\mathrm{j}$ Xij sample observation value, to evaluate the corresponding object I and diagnosis. Specific steps are as follows:

1) According to the qualitative analysis, to determine operational performance index system and weight of each index in the comprehensive clustering.

2) depict grey class according to requirements of the assessment, selection of threshold. Set in accordance with the requirements for evaluation of ash class number of $S$, the scope of each index is correspondingly divided into $\mathrm{S}$ a grey class, the scope of such as $\mathrm{j}$ index [a1, as +1 ] divided into/a1, $\mathrm{a} 2, \ldots$ [ak - 1, ak],... ., [as] the as - 1, and [the as, as +1 ]. Among them: the threshold ak $(\mathrm{k}=1,2$, $3, \ldots$, s) value, can according to the requirement of the practical problems or qualitative research results.

3) establish a triangular whitenization weight function. To belong to the first k grey whitenization weight function of a class value is 1 , and (1) and the start of the first $\mathrm{k}-1$ grey class and the end of the first $\mathrm{k}+1$ grey class connection, thus can get $\mathrm{j}$ index of $\mathrm{k}$ grey class

Triangular whitenization weight function $\mathrm{j}=1,2,3, \ldots \mathrm{M} ; \mathrm{K}=1,2,3, \ldots \mathrm{M} ; \mathrm{K}=1,2,3, \ldots$, s. To and can be respectively according to the particular case according to the specific situation will $\mathrm{j}$ index number field to the left and right along the extension to a0, As +2 , as shown in figure 1 .

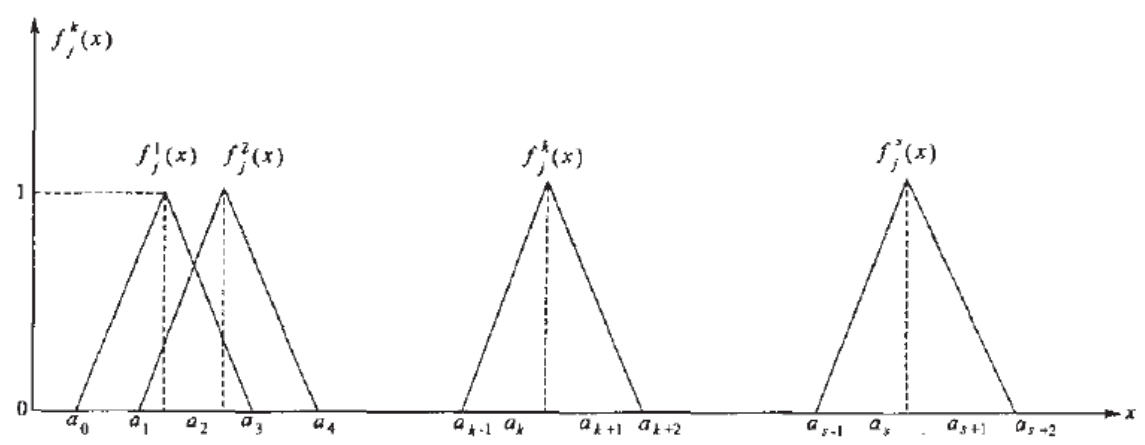

Fig.1 Triangular whitenization weight function

4) to sort out the indexes before and after modification to realize value, it belongs to the grey class are calculated separately, and the membership degree. For an implementation value $\mathrm{x} j \mathrm{j}$ index, the weight function formula

$$
\mathrm{f}_{\mathrm{j}}^{\mathrm{k}}(\mathrm{x})=\left\{\begin{array}{c}
0, \quad \mathrm{x} \notin\left[a_{k-1}, a_{k+2}\right] \\
\left(x-a_{k-1}\right) /\left(\lambda_{k}-a_{k-1}\right), x \in\left[a_{k-1}, \lambda_{k}\right] \\
\left(a_{k+2}-x\right) /\left(a_{k+2}-\lambda_{k}\right), x \in\left[\lambda_{k}, a_{a+2}\right]
\end{array}\right.
$$

Calculate the belongs to the grey class $k(k=1,2,3, \ldots s)$ membership degree, one of them 
5) comprehensive clustering coefficient calculation, the results of the analysis, make reasonable judgment.

(1) calculate the object $(I=1,2,3, \ldots, n)$ breakdown performance indicators, such as fire, move, protection, communication skills, and operational performance of grey class $k(k=1,2,3, \ldots s)$ clustering coefficient $\delta_{\mathrm{i}}^{\mathrm{k}}$.

$\delta_{i}^{k}=\sum_{j=j 1}^{j 2} f_{j}^{k}\left(x_{i j}\right) \bullet \eta_{j}$

Type: $\left(\mathrm{f}_{\mathrm{j}}^{\mathrm{k}}\left(\mathrm{x}_{\mathrm{ij}}\right)\right.$ ) as the object under the index $\mathrm{j}$ belong to the grey class $\mathrm{k}$ albino functions; For $\mathrm{j}$ in

$\eta_{j}$ comprehensive clustering weights (all comprehensive weights is 100); [j1, j2] as the index range includes a child of the indicators.

(2) by judging the object I belong to the grey class $\mathrm{k}$ *

When there are multiple objects belong to $\mathrm{k} *$, can further according to the grey clustering to determine the size of the integrated belong to k class of the pros and cons of each object or places to make reasonable judgment of comprehensive performance, to determine the need to repack.

\section{Military Operational Requirements Analysis}

\section{Operational Requirements Analysis.}

Imagine new anti-ship amphibious armored vehicles as mobility of the south China sea reef guard amphibious landing operations I ShouDao troops equipped with attachment. So I ShouDao forces on the south China sea reefs have a powerful mobile amphibious armored vehicle power, and small and medium-sized ships with an incoming enemy formation on the sea, for an amphibious landing islands and waters. In formation against the small and medium-sized ship suddenly hit me in the south China sea islands, I ShouDao forces using new anti-ship amphibious armored vehicles to amphibious landing operations against the enemy of the invasion, persevere until I arrive naval and air support, which achieved the purpose of protect maritime territory. If I ShouDao forces only for infantry, anti lagunas raid combat only in island $\mathrm{j} 2$, land area only islands itself, the enemy of the invasion will focus on ship formation narrow land on fire I reef defenders. If I ShouDao troops equipped with new anti-ship amphibious armored vehicles, flood water in new anti-ship amphibious armored vehicles navigation mobility and firepower attack (distance), against anti-ship amphibious landing area will enlarge the hundreds of thousands of times, against anti-ship amphibious landing firepower and combat ability will be greatly enhanced, this is my ShouDao forces against landing operations weapons and equipment foundation to success.

\section{New Anti-ship Deployment Problem of Amphibious Armored Vehicles.}

New anti-ship amphibious armored vehicles based on south China sea islands, saved, due to the reason of the oceanic climate, equipment may rust the land quickly. Based on the reef environmental conditions, the vehicle maintenance may be more difficult, besides wanting to notice at ordinary times maintain ShouDao forces and anti-corrosion, amphibious armored vehicles as long as the regular evacuation maintenance or organization level relay circuit maintenance can solve the problem. May be considered in hainan port bases or three shashi to build amphibious armored vehicle maintenance base, amphibious armored vehicles stationed in the south China sea islands, saved rotate back to base on a regular basis for equipment maintenance, to ensure the integrity of equipment and operational capability.

\section{Protection and Stealth.}

New anti-ship amphibious armored vehicle targets on the sea is very small, reveal about the height of the surface but $\mathrm{lm}$ and along with the wave of ups and downs, itself is not a harpoon, flying fish within the scope of anti-ship missile attack. But in order to prevent by investigation and lock, still need to new anti-ship amphibious anti-ship armored vehicles stealth protection measures.

1) with low radar scattering cross section RCS (radar cross - section, shape design and contour 
integration design (exposed equipment and bodywork and turret reasonable fusion).

2) structure of stealth technology, such as the vehicle to reduce the infrared radiation of the structure, shielding and noise reduction structure, power capsule and exhaust pipe insulation and reduce infrared radiation measures, smoke is cooled and diluted mixed after discharge, body spray can prevent optical, radar, infrared, millimeter-wave reconnaissance multifunctional composite coating, the vehicle of water curtain and water mist stealth measures, etc.

3) local use stealth camouflage nets.

4) partial use stealth function of wear armor.

\section{Conclusion}

In recent years, the gray clustering evaluation method applied in various fields has larger development, this paper applied in amphibious armored vehicle operational performance evaluation before and after modification, gives the general steps of the assessment, through concrete example analysis, better solve the problem of the performance of the equipment before and after modification contrast analysis, provides reference for the future of amphibious armored equipment improvement. Our country ShouDao disposition can choose the type of defense weapons are: a navy destroyer and frigate, new anti-ship amphibious armored vehicles, army general firepower system. Larger army conventional firepower system limitations, the first is to deploy is difficult, and in Marine amphibious reef defensive operations cannot be moved, battle damage probability is big. Destroyer and frigate self sustaining cycle is $30 \sim 45$ days commonly, defend the reef group of several ships take turns on duty, deployed ships defend reef, cost is higher, the warship for patrol and instant support. Look at new anti-ship amphibious armored vehicles, easy deployment, amphibious performance is good, anti-ship landing operations capability is strong, amphibious the chances of survival, shallow sea, good adaptability to the environment, dozens of square meters of reef can be parked. Namely or shallow reef through general geotechnical construction of parking Spaces, can also be parked, meet long-term garrison reef effective practical control sea land requirements, cost is relatively small, so the new anti-ship amphibious armored vehicles is the most appropriate reef defense weapons and equipment. In the south China sea reef garrison of amphibious armored vehicles equipped with new anti-ship ShouDao forces is the most suitable operational requirements.

\section{References}

[1]Nanrong Xu,Weijun Zhong. Scientific decision-making theory and method[M],Nanjing: Southeast university press,1995.

[2]Zhanping Zhang,Zhengxin Yang. 122 truck-mounted howitzer ChanBao operational capability analysis[J]. Journal of shooting, 2005(2),9-11.

[3] Ping He. The Vietnamese navy[J].Weapon knowledge,2008(1):33-36. 